\title{
Studying Host-Virus Alliances using CRISPR Genetics
}

\author{
Rashmi Dahiya and Md. Imtaiyaz Hassan* \\ Center for Interdisciplinary Research in Basic Sciences, India
}

Submission: April 24, 2017; Published: June 20, 2017

*Corresponding author: Md Imtaiyaz Hassan, Assistant Professor, Center for Interdisciplinary Research in Basic Sciences, Jamia Millia Islamia, New Delhi 110025, India, Email: mihassan@jmi.ac.in

Keywords: Pamp; Dsb; Crispr-Cas; Nhej; Cmas; Npc1; Lamp1; Npc1; Erad; Ost; Trap

\section{Introduction}

Viruses are lifeless, obligate, intracellular particles that serve as a carrier for their own genome, and require host cell components for replication. Through cell surface receptors these pathogens enter cells and adapt themselves with the host cellular machinery. Host cells, in turn, can neutralize the infection by sensing Pathogen-Associated Molecular Patterns (PAMPs), prompting the expression of antiviral proteins. The identification and characterization of these antiviral factors can provide ground-breaking insights into the host-virus alliance and basic facets of molecular biology leading towards discovery of potential targets in antiviral therapeutics. Approaches like forward genetic screening has markedly enhanced our understanding of fundamental biological processes in genetically tractable models like yeasts, drosophila, zebrafish and worms, however, these approaches were limited to these organisms. Due to the experimental complications, implicating forward genetics to cultured mammalian cells is a daunting and challenging task.

Interestingly, in mammalian systems, loss of function genetic approaches has lagged behind the gain of function approaches merely due to the scarcity of molecular tools that could function in high-throughput manner targeting both allele's indiploid genomes. Vector based ectopic expression of desired genes as a gain of function approach has been very successful in mammalian cells. Indeed, using this technique, various host cell surface receptors required for virus entry has been identified. Simply put, cell lines which are not susceptible for viral infection are transformed with cDNA libraries of viral infection permissive cell lines. The Hepatitis C viral entry through claudin 1 and occluding receptors were identified using cDNA libraries from hepatocellular carcinoma cells. In contrast, loss of function approaches required stable knockout or knockdown of target loci. Initially RNAi approach has advanced our understanding of host-viral relationships [1,2]. Later on, a technique termed as haploid genetic screening had emerged which exploited the haploid human cell lines through insertion mutagenesis. Nearhaploid karyotype of chronic myeloid cell line KBM-7 cells has been exploited to perform large-scale loss-of-function screens in human cells $[3,4]$.

This technique used gene-trap retroviruses containing a strong adenoviral splice variant site and a marker gene for selection. Insertional mutagenesis approach is highly mutagenic in a variety of organisms which also integrate a molecular tag for identification of disrupted gene. Using this approach, an enzyme known as cytidine monophosphate $\mathrm{N}$-acetylneuraminic acid synthase (CMAS) and a transporter protein SLC35A2 were identified to be required for influenza virus infection. Indeed, mutants of these two genes showed complete resistance to influenza [4].

Later on, haploid genetic screen has identified Niemannpick C1 protein (NPC1) as a potential receptor for Ebola virus glycoprotein [5]. Interestingly, mutations were identified in the Ebola glycoprotein during 2013-2016 Ebola outbreaks which were found to increase the infection of primates [6]. In addition, Lassa viral glycoprotein was found to interact with Lysosome Associated Membrane Glycoprotein 1 (LAMP1) [7]. Indeed, Technological advances like RNAi and insertion mutagenesis had eased the task of disrupting gene expression in mammalian cells on a large genomic scale. However, RNAi knockdown approaches 
results in variable levels of target gene depletion and Haploid genetic screens are confined to specific cell types containing a haploid karyotype.

Recently, the discovery of CRISPR/Cas genome editing approach has revolutionized the field of biological research. The reproducibility and target selectivity's of CRISPR/Cas screens have surpassed the genetic screen performed by RNAi. CRISPR-Cas Knock-out alleles results in marked phenotypes and lower number of false positives. Interestingly, the initial discoveries using KBM7 cells revealed that it contained disomy of chromosome 8 and a portion of chromosome 15. However HAP1, a derivative of KBM7 cells have generated through genetic reprogramming, lacks second copy of chromosome 8. However, they are not complete haploid since they have retained a fragment of chromosome 15 fused to chromosome 19. Interestingly, CRISPR/Cas9 system was employed to delete Chromosome 15 disomy by deleting $\sim 30$ million bases, encoding 330 human genes [8].

Viruses infect host in broad cell specific range which limits the haploid screening in potential identification of pathogenic factors. Virus permissive or non-permissive cells further limit the efficacy of haploid screening. In contrast, CRISPR/Cas genome editing has been conducted in a wide range of cell lines offering best results. Conventionally, CRISPR/Cas employed an endonuclease, Cas9, directed towards a specific genomic location by a single guide RNA (sgRNA) through base pairing. Cas9 generates DSB (Double Strand Break) at the target site which is then repaired by NHEJ (Non-Homologous End Joining), which often resulting in frame shift mutations expressing nonfunctional proteins. Successive reports have published in peer journals regarding the genome-wide identification of host components required for virus replication. This formidable task became possible with the ease of Cas9 specific targeting to different loci, using numerous sgRNA pools spanning entire human genome. CRISPR sgRNA libraries are available in Add gene repositories covering target gene and targeted positions within genes. Initial genome wide CRSIPR knockout libraries (GeCKO) contained 4-6 sgRNA/gene. To minimize off-target effect and statistical enrichment of target locations it was further refined up to $12 \mathrm{sgRNA} /$ gene $\mathrm{t}$ [9-11].

The potential roles of CRISPR/Cas in discovering host virus relationships are numerous in numbers, leading towards the development of antiviral therapeutics. Several viruses have been studied using CRSIPR/Cas screening. Mosquito-borne virus DENV (Dengue Virus), ZIKV (ZIka virus), WNV (West Nile Virus) and Chikungunya virus have been characterized on the basis of their specificities in host interactions. A number of ER proteins are identified required for virus replications. Specifically, the components of ER-associated protein degradation pathway (ERAD, oligosaccharyl transferase complex (OST) and translocon-associated protein (TRAP) complex have been identified using CRISPR technology. Remarkably, flavivirus replication decreased up to 10,000 fold upon knocking out those enriched proteins $[12,13]$. CRISPR/Cas screening of CD4+T cell lines have identified host factors required for HIV replication. Characterization of CD4 and co-receptor CCR5 (Chemokine receptor 5), CD166 and SLC35B2 transporter was achieved through CRISPR pathways in HIV infection [14]. Apart from that, mechanisms of host entry and cellular receptors of Noroviruses, a leading cause of gastroenteritis globally has been identified as well. CD300LF also known as CLM1, a protein receptor has been identified for norovirus infection through CRISPR/Cas screens. Genome editing of large DNA viruses like Epstein-Barr virus, Vaccinia virus and adenoviruses has been efficiently performed using CRISPR mechanism [15].

Additionally, mechanism of action of antiviral drugs like GSK983 has been elucidated by CRISPR/Cas which inhibits viral replication through inhibition of dihydroorotatedehydrogenase, an enzyme required for pyrimidine biosynthesis. It has been further uncovered that GSK983 reduce nucleotide levels through enzyme inhibition thereby blocking viral DNA synthesis [16]. Since its discovery, the repurposing of CRISPR/Cas mechanism as a potential genetic engineering tool has completely transformed the biological research in all aspects such as gene therapy, cancer and pathogenesis of various infectious diseases. The on-going efforts to further development and enhancement of CRISPR/ Cas system will help us to eradicate current and future diseases. Furthermore, combination of other genetic tools with CRISPR/ Cas will uncover functional interdependencies of viral and host mutualism. We believe that expansion and refinement of this genetic tool for mammalian host cells manipulation will lead to the end of cold war between host and viruses.

\section{References}

1. Ramage H, Cherry S (2015) Virus-Host Interactions: From Unbiased Genetic Screens to Function. Annu Rev Virol 2(1): 497-524.

2. Paddison PJ, Silva JM, Conklin DS, Schlabach M, Li M, et al. (2004) A resource for large-scale RNA-interference-based screens in mammals. Nature 428(6981): 427-431.

3. Kotecki M, Reddy PS, Cochran BH (1999) Isolation and characterization of a near-haploid human cell line. Exp Cell Res 252(2): 273-280.

4. Carette JE, Guimaraes CP, Varadarajan M, Park AS, Wuethrich I, et al. (2009) Haploid genetic screens in human cells identify host factors used by pathogens. Science 326(5957): 1231-1235.

5. Carette JE, Raaben M, Wong AC, Herbert AS, Obernosterer G, et al. (2011) Ebola virus entry requires the cholesterol transporter Niemann-Pick C1. Nature 477(7364): 340-343.

6. Diehl WE, Lin AE, Grubaugh ND, Carvalho LM, Kim K, et al. (2016) Ebola Virus Glycoprotein with Increased Infectivity Dominated the 2013-2016 Epidemic. Cell 167(4): 1088-1098.

7. Jae LT, Raaben M, Herbert AS, Kuehne AI, Wirchnianski AS, et al. (2014) Virus entry. Lassa virus entry requires a trigger-induced receptor switch. Science 344(6191): 1506-1510.

8. Essletzbichler P, Konopka T, Santoro F, Chen D, Gapp BV, et al., Megabasescale deletion using CRISPR/Cas9 to generate a fully haploid human cell line. Genome Res 24(12): 2059-2065. 
9. Sanjana NE, Shalem O, Zhang F (2014) Improved vectors and genomewide libraries for CRISPR screening. Nat Methods 11(8): 783-784.

10. Shalem O, Sanjana NE, Hartenian E, Shi X, Scott DA, et al. (2014) Genome-scale CRISPR-Cas9 knockout screening in human cells. Science 343(6166): 84-87.

11. Doench JG, Fusi N, Sullender M, Hegde M, Vaimberg EW, et al. (2016) Optimized sgRNA design to maximize activity and minimize off-target effects of CRISPR-Cas9. Nat Biotechnol 34(2): 184-191.

12. Marceau CD, Puschnik AS, Majzoub K, Ooi YS, Brewer SM, et al. (2016) Genetic dissection of Flaviviridae host factors through genome-scale CRISPR screens. Nature 535(7610): 159-163.
13. Zhang R, Miner JJ, Gorman MJ, Rausch K, Ramage H, et al. (2016) A CRISPR screen defines a signal peptide processing pathway required by flaviviruses. Nature 535(7610): 164-168.

14. Park RJ, Wang T, Koundakjian D, Hultquist JF, Lamothe-Molina P, et al. (2017) A genome-wide CRISPR screen identifies a restricted set of HIV host dependency factors. Nat Genet 49(2): 193-203.

15. Tan M, X Jiang (2005) Norovirus and its histo-blood group antigen receptors: an answer to a historical puzzle. Trends Microbiol 13(6): 285-293.

16. Deans RM, Morgens DW, Ökesli A, Pillay S, Horlbeck MA, et al. (2016) Parallel shRNA and CRISPR-Cas9 screens enable antiviral drug target identification. Nat Chem Biol 12(5): 361-366.

\section{Your next submission with Juniper Publishers} will reach you the below assets

- Quality Editorial service

- Swift Peer Review

- Reprints availability

- E-prints Service

- Manuscript Podcast for convenient understanding

- Global attainment for your research

- Manuscript accessibility in different formats

( Pdf, E-pub, Full Text, Audio)

- Unceasing customer service

Track the below URL for one-step submission https://juniperpublishers.com/online-submission.php 